\title{
Spin Degree of Freedom in Two Dimensional Exciton Condensates
}

\author{
J. Fernández-Rossier and C. Tejedor \\ Departamento de Física Teórica de la Materia Condensada, Universidad Autónoma de Madrid, Cantoblanco, 28049 Madrid, Spain
} (Received 23 December 1996)

\begin{abstract}
We present a theoretical analysis of a spin-dependent multicomponent condensate in two dimensions. The case of a condensate of resonantly photoexcited excitons having two different spin orientations is studied in detail. The energy and the chemical potentials of this system depend strongly on the spin polarization. When electrons and holes are located in two different planes, the condensate can be either totally spin polarized or spin unpolarized, a property that is measurable. The phase diagram in terms of the total density and electron-hole separation is discussed. [S0031-9007(97)03434-0]
\end{abstract}

PACS numbers: 71.35.Lk

In this Letter we address the problem of a multicomponent quantum liquid: a condensate of excitons which hold an internal degree of freedom, namely, the third component of the angular momentum $M$ [1]. The multicomponent character is twofold: on one side, the condensate is formed by pairs of distinct particles, an electron and a hole; on the other side, more than one kind of pair exists when $M$ is taken into account. From the later point of view, there is an evident analogy with some of the superfluid ${ }^{3} \mathrm{He}$ phases in which a condensate with two components appears due to triplet pairing [2]. We consider excitons generated in quasi-two-dimensional (2D) semiconductor heterostructures where carriers' lifetimes can be very large. Moreover, degeneracy at the top of the valence band is broken usually leaving the highest hole with a third component of the angular momentum equal to $\pm 3 / 2$. Then, four types of excitons with different values of $M$ exist, implying that different components of the condensate with different populations $N_{M}$ for each spin are possible. The condensate properties depend on both the total density and relative populations. Hence, the problem offers more possibilities than the spinless exciton case considered in the literature [3-9]; e.g., Josephson-type effects between the different components of the condensate [10] are now possible.

We have two main reasons for paying attention to $M$ :

(i) The first is related to experiments. A promising way to obtain a multicomponent exciton condensate is to excite the semiconductor with circularly polarized light from a pulsed laser. Because of the angular momentum selection rule, two different kinds of excitons can be created, with either $M=+1$ or -1 . The light polarization produces a spin polarization $P=\left(N_{+}-N_{-}\right) /\left(N_{+}+N_{-}\right)$[11], where $N_{ \pm}$are the populations of excitons with $M= \pm 1$, respectively $[12,13]$. It is interesting to study the properties of the photoexcited carriers with unbalanced populations $N_{M}$ by time-resolved photoluminescence. There are time evolutions both on the total density due to recombination and on $P$ due to spin relaxation processes. Frequency and intensity of the emitted photons with the two different circular polarizations allow the experimental study of the condensates. It is possible to observe how the chemical potentials of each kind of excitons in the condensate are different; the splitting of the exciton levels turns out to be strongly dependent on $P$ [13].

(ii) The second reason is related to the physical origin of the existence of different phases and is precisely the aim of this Letter. Each phase is characterized by different populations of each component of the condensate; e.g., in the case of a condensate with two components with $M=+1$ and $M=-1$, respectively, it can be either a ferromagnetic phase with $N_{+}=N$ and $N_{-}=0$ or a paramagnetic phase with $N_{+}=N_{-}=N / 2$ [11]. The reason for the existence of different phases is the exclusion principle which plays a crucial role in the interaction between excitons in semiconductors. Effective masses are very light so that electron and hole wave functions are very extended implying large overlaps which produce highly spin-dependent effective interactions between excitons. We will show that the main consequence of this is that the ground state can be either spin polarized or spin unpolarized. This depends on both the carrier density and the relative intensities of attractive and repulsive interactions that can be controlled by spatially separating electron and hole gases with respect to each other.

Our target is to study the ground state of $N$ electrons and $N$ holes lying in two different planes separated by a distance $d$. The Hamiltonian is given by

$$
\begin{aligned}
H= & \sum_{\mathbf{k} \boldsymbol{\sigma}}\left(\varepsilon_{\mathbf{k}}^{e} a_{\mathbf{k} \boldsymbol{\sigma}}^{\dagger} a_{\mathbf{k} \boldsymbol{\sigma}}+\sum_{\mathbf{k}^{\prime}, \mathbf{q}, \sigma^{\prime}} \frac{V_{\mathbf{q}}^{f f}}{2 L^{2}} a_{\mathbf{k} \boldsymbol{\sigma}}^{\dagger} a_{\mathbf{k}^{\prime} \boldsymbol{\sigma}^{\prime}}^{\dagger} a_{\mathbf{k}^{\prime}-\mathbf{q} \sigma^{\prime}} a_{\mathbf{k}+\mathbf{q} \sigma}\right) \\
& +\sum_{\mathbf{k} \mathbf{s}}\left(\varepsilon_{\mathbf{k}}^{h} b_{\mathbf{k} \boldsymbol{s}}^{\dagger} b_{\mathbf{k} \boldsymbol{s}}+\sum_{\mathbf{k}^{\prime}, \mathbf{q}, \mathbf{s}^{\prime}} \frac{V_{\mathbf{q}}^{f f}}{2 L^{2}} b_{\mathbf{k} \boldsymbol{s}}^{\dagger} b_{\mathbf{k}^{\prime} \mathbf{s}^{\prime}}^{\dagger} b_{\mathbf{k}^{\prime}-\mathbf{q} \boldsymbol{s}^{\prime}} b_{\mathbf{k}+\mathbf{q} \boldsymbol{s}}\right) \\
& +\sum_{\mathbf{k}, \mathbf{k}^{\prime}, \mathbf{q}, \boldsymbol{\sigma}, \boldsymbol{s}} \frac{V_{\mathbf{q}}^{e h}(d)}{L^{2}} a_{\mathbf{k} \boldsymbol{\sigma}}^{\dagger} b_{\mathbf{k}^{\prime} \mathbf{s}}^{\dagger} b_{\mathbf{k}^{\prime}-\mathbf{q} \boldsymbol{s}} a_{\mathbf{k}+\mathbf{q} \sigma}
\end{aligned}
$$

where $\mathbf{k}, \mathbf{k}^{\prime}$, and $\mathbf{q}$ are 2D wave vectors, $\sigma$ stands for the electron spin $\pm 1 / 2(\uparrow, \downarrow)$ and $s$ for the hole spin $\pm 3 / 2$ $(\Uparrow, \downarrow)$ along the direction perpendicular to the $2 \mathrm{D}$ plane. Creation and annihilation operators are labeled as $a$ 's for electrons and $b$ 's for holes with single particle energies $\varepsilon_{\mathbf{k}}^{e}$ 
and $\varepsilon_{\mathbf{k}}^{h}$, respectively. $L^{2}$ is the area of the sample and the interactions $V_{\mathbf{q}}^{e h}(d)$ and $V_{\mathbf{q}}^{f f}(f \equiv e, h)$ will be specified below.

It is convenient to point out some assumptions behind this Hamiltonian. First of all, we suppose that the electron-hole $(e-h)$ spatial degrees of freedom are in thermodynamic equilibrium. This hypothesis is supported by the fact that roughly $1 \mathrm{ps}$ after the photoexciting laser pulse has finished, the $e-h$ liquid reaches a thermal momentum distribution [14]. That distribution corresponds to zero temperature when experiments are performed in the resonant excitation regime. Since recombination and spin relaxations are processes much slower than that thermalization $[12,13]$, we take both the carrier density and the spin polarization as external parameters in the theory, in the spirit of a Born-Oppenheimer framework.

In order to look for exciton condensates we use a mean field Keldysh-Kopaev formalism, i.e., a generalization of the BCS treatment $[3,5,6]$. We define two propagators $G$ for each fermion, and four anomalous propagators $F$ for the four pairings $(\uparrow, \Uparrow),(\downarrow, \downarrow),(\uparrow, \downarrow),(\downarrow, \Uparrow)$ :

$$
\begin{aligned}
G_{\sigma}^{e}(k, t) & =-i\left\langle T a_{\mathbf{k}, \boldsymbol{\sigma}}(t) a_{\mathbf{k}, \boldsymbol{\sigma}}^{\dagger}(0)\right\rangle, \\
G_{\mathrm{\varsigma}}^{h}(k, t) & =-i\left\langle T b_{\mathbf{k}, \boldsymbol{s}}(t) b_{\mathbf{k}, \boldsymbol{s}}^{\dagger}(0)\right\rangle, \\
F_{\sigma \boldsymbol{\varsigma}}^{e h}(k, t) & =-i\left\langle T a_{\mathbf{k}, \boldsymbol{\sigma}}(t) b_{\mathbf{k}, \boldsymbol{s}}(0)\right\rangle .
\end{aligned}
$$

Each one of the anomalous propagators is associated with a different type of $e-h$ pair $(\sigma, \varsigma)$ and with a gap (order parameter of the condensate) $\Delta_{\sigma \varsigma}(k)=$ $\sum_{\mathbf{q}} V_{|\mathbf{k}-\mathbf{q}|}^{e h}\left\langle T a_{\mathbf{q}, \boldsymbol{\sigma}}(0) b_{\mathbf{q}, \mathbf{s}}(0)\right\rangle$ proportional to the density of $e$ - $h$ pairs $(\sigma, \varsigma)$. Following a standard mean field procedure one obtains the propagators [10]

$$
G_{\sigma}^{e}(k, \omega)=\frac{\Omega_{\Downarrow}^{h} \Omega_{\Uparrow}^{h}}{\Omega_{\sigma}^{e} \Omega_{\Downarrow}^{h} \Omega_{\Uparrow}^{h}+\Omega_{\Downarrow}^{h} \Delta_{\sigma \Uparrow}^{2}(k)+\Omega_{\Uparrow}^{h} \Delta_{\sigma \Downarrow}^{2}(k)},
$$

$$
F_{\sigma, \mathbf{S}}^{e h}(k, \omega)=\frac{-\Delta_{\sigma \boldsymbol{\varsigma}}(k) \Omega_{-\varsigma}^{h}}{\Omega_{\sigma}^{e} \Omega_{\Downarrow}^{h} \Omega_{\Uparrow}^{h}+\Omega_{\Downarrow}^{h} \Delta_{\sigma \Uparrow}^{2}(k)+\Omega_{\Uparrow}^{h} \Delta_{\sigma, \Downarrow}^{2}(k)} .
$$

For simplicity we have not written the hole propagator, $G_{\mathrm{s}}^{h}(k, \omega)$, which has a similar expression to $G_{\sigma}^{e}(k, \omega)$ simply exchanging $e$ with $h . \quad \Omega_{\mathrm{s}}^{h}(k, \omega)=$ $-i \omega-\left[\varepsilon_{\mathbf{k}}^{h}-\mu_{\mathrm{s}}^{h}-\Sigma_{\mathrm{s}}^{h}(k)\right], \quad \Omega_{\sigma}^{e}(k, \omega)=-i \omega+$ $\varepsilon_{\mathbf{k}}^{e}-\mu_{\sigma}^{e}-\sum_{\sigma}^{e}(k)$ with $\mu_{\sigma}^{e}$ and $\mu_{\mathrm{s}}^{h}$ being electron and hole chemical potentials and $\Sigma$ is the usual Hartree-Fock fermion self-energy.

In the expressions for the propagators one observes the difference between the condensate we are studying and those occurring in other problems like superconductors, ${ }^{3} \mathrm{He}$ superfluid, or the spinless exciton gas. Since the pairing occurs among two different types of fermions, each one with different spin, the terms $\Omega_{\sigma}^{e} \Omega_{\mathrm{s}}^{h} \Omega_{-{ }_{\varsigma}}^{h}$ appearing in the denominator produce three poles for each propa- gator. This causes a complicated mixing of excitations unlike other types of condensates [15].

In this paper we will focus on the case of resonant excitation of $(\downarrow, \Uparrow)$ pairs. Under resonance conditions, holes do not relax their spin independently $[12,16]$, a process which should produce $(\downarrow, \Downarrow)$ pairs with $M=$ -2 . Instead, the main effect is a relaxation of the angular momentum of the whole exciton, by means of an intraexciton exchange spin flip, from $M=+1$ to $M=-1[12,16]$. Therefore, we neglect the densities of excitons with both $M= \pm 2$ and only two kinds of excitons are considered: those with $M=-1 \equiv(\uparrow, \downarrow)$ and those with $M=+1 \equiv(\downarrow, \Uparrow)$ having densities $n_{-}=$ $N_{-} / L^{2}$ and $n_{+}=N_{+} / L^{2}$, respectively. Such a system will be characterized by two quantities, the total density $n=N / L^{2}$ and the polarization $P$. In this case, either the second or the third term in the denominators of Eqs. (3) and (4) disappears, one of the $\Omega^{h}$ 's can be canceled out, and each propagator only has two poles as in the BCS or superfluid ${ }^{3} \mathrm{He}$ theory. Then one could follow standard procedures [10] to obtain gap equations for $\Delta_{\downarrow, \Uparrow}(k)$ and $\Delta_{\uparrow, \Downarrow}(k)$ [hereafter denoted as $\Delta_{+}(k)$ and $\Delta_{-}(k)$, respectively] which are coupled to each other solely through the Hartree contribution which is spin independent [15]. For an homogeneous system, the Hartree term can be embedded in the chemical potential as an energy origin so that the gap equations become decoupled to all the effects. Then, solving these gap equations is equivalent to looking for an extremal from

$$
\left\langle\Psi\left|H-\mu_{+} N_{+}-\mu_{-} N_{-}\right| \Psi\right\rangle,
$$

using a wave function

$$
\begin{aligned}
|\Psi\rangle= & \prod_{\mathbf{k}, \mathbf{k}^{\prime}}\left(u_{k}^{+}+v_{k}^{+} a_{\mathbf{k} \downarrow}^{\dagger} b_{\mathbf{k} \Uparrow \Uparrow}^{\dagger}\right) \\
& \times\left(u_{k^{\prime}}^{-}+v_{k^{\prime}}^{-} a_{\mathbf{k}^{\prime} \uparrow}^{\dagger} b_{\mathbf{k}^{\prime} \Downarrow}^{\dagger}\right)|0\rangle .
\end{aligned}
$$

$\mu_{ \pm}$are the chemical potentials of \pm 1 excitons and $|0\rangle$ is the ground state of the system without any photoexcited $e-h$ pairs. Each set of parentheses in Eq. (6) corresponds to one of the two components of the condensate. As in the spinless case, this wave function interpolates smoothly between two limits, $n \rightarrow 0$ and $n \rightarrow \infty$, where it is exact [5]. The normalization condition $\langle\Psi \mid \Psi\rangle=1$ of the condensate wave function imposes the constraints $\left(u_{k}^{ \pm}\right)^{2}+\left(v_{k}^{ \pm}\right)^{2}=1$. The trial wave function (6) is valid under resonance conditions, as discussed above, and it leads to a gap equation $\Delta_{ \pm}(k)=\sum_{\mathbf{q}} V_{|\mathbf{k}-\mathbf{q}|}^{e h} u_{q}^{ \pm} v_{q}^{ \pm}$. The practical procedure is to fix the two chemical potentials $\mu_{ \pm}$and to minimize the functional (5) in terms of the parameters $u_{k}^{ \pm}, v_{k}^{ \pm}$in a discretized $k$ space. Since the Coulomb interaction is singular in $2 \mathrm{D}$, we follow the usual procedure [6] of adding and subtracting a term that makes the singularity analytically integrable. From $u_{k}^{ \pm}, v_{k}^{ \pm}$we get $\Delta_{ \pm}(k)$ and the density, $n_{ \pm}=\sum_{k}\left(v_{k}^{ \pm}\right)^{2}$, for each type of $e$-h pair. So we have all the ingredients to obtain the Green functions, the energy per pair, etc. Changing $\mu_{ \pm}$ 
we can obtain the dependence of the energy per pair for each spin on both $n$ and $P$. The results so obtained for $\mu_{ \pm}$as a function of $n$ and $P$ are directly comparable with photoluminescence experiments which measure the energy liberated when an exciton recombines [12,13].

Let us discuss the physics involved in our mean field theory. We will not include screening which does not depend on $P$ when treated to the lowest order in perturbation theory [17], and it does not affect the splitting between chemical potentials $\delta=\mu_{+}-\mu_{-}$which is our main interest in this paper. In this approach there are three many-body contributions to the renormalization of the single exciton chemical potential: The first is a Hartree term which gives the unique coupling between the two components of the condensate. The other two contributions are related to the Pauli principle. One is the exchange correction (EC), i.e., the reduction of the repulsive electron-electron (hole-hole) interaction due to the fact that two identical fermions cannot occupy the same state. The last contribution is the vertex correction (VC), which represents the reduction of the $e-h$ attraction due to the occupation of final states in $e-h$ scattering processes. $\mathrm{EC}$ and $\mathrm{VC}$ contributions to the chemical potential of a fermion with a given spin are independent of the amount of fermions with the opposite spin. This causes a nonzero splitting, $\delta$, whenever there is a nonzero spin polarization $P$, in agreement with experiments $[12,13]$. All these physical properties are described by a wave function (6) for the condensate which is separable in two components in analogy to what happens in the A phase of superfluid ${ }^{3} \mathrm{He}$ due to equal spin pairing (ESP) states [2]. The exciton condensate that we present in this paper is more flexible than ESP states of ${ }^{3} \mathrm{He}$ because $P$ and $\delta$ can be nonzero in the exciton case, while they must be zero in the ESP case.

The numerical results shown in this paper are obtained with $V_{\mathbf{q}}^{e h}(d)=-e^{2} \exp (-q d) / \epsilon q$, i.e., the Fourier transform of $V^{e h}(r, d)=-e^{2} / \epsilon \sqrt{r^{2}+d^{2}}$, and $V \mathbf{q}^{f f}=$ $-V_{\mathbf{q}}^{e h}(d=0), \epsilon$ being the dielectric constant of the material. The single particle energy bands are supposed to be isotropic and parabolic. The spinless case has been studied by Zhu et al. [8], who considered $d \neq 0$ because the $e-h$ separation increases the exciton lifetime improving the condensation conditions and avoids formation of biexcitons. We have an additional reason to be interested in the case $d \neq 0: d$ is a parameter which controls the relative strengths of $\mathrm{EC}$ and $\mathrm{VC}$ which compete with each other. Hence, by tuning $d$ and $n$, one can obtain a quantum phase transition between a ferromagnetic phase (dominated by exchange) and a paramagnetic phase (dominated by the vertex corrections). This is a crucial result coming out from our study of the spin degree of freedom in the exciton condensate.

For all the results presented in this paper we scale the length to the three-dimensional Bohr radius $a=$ $\hbar^{2} \epsilon / m^{*} e^{2}$, where $m^{*}=m^{e} m^{h} /\left(m^{e}+m^{h}\right)$ is the exciton reduced mass. Energies are taken with origin at the bottom of the conduction band and are given in units of the 2D Rydberg $\left|E_{0}^{2 \mathrm{D}}\right|=2 e^{2} / \epsilon a$.

Figure 1 shows chemical potentials $\mu_{ \pm}$as a function of the density for $P=0.8$ and three values of the separation $d$. $\mu_{ \pm}$are always increasing with $n$ because excitonexciton interaction turns out to be globally repulsive. That means that repulsive Hartree and VC terms in the energy dominate on the attractive EC. The essential point is the behavior of $\delta=\mu_{+}-\mu_{-}$, which is a decreasing function of $d$ for moderate densities, and it can be positive or negative. This is shown more clearly in the inset. The first conclusion is that $\delta$ is in the range of $\mathrm{meV}$ and is consequently an experimentally accessible quantity [12,13]. Since the Hartree term is spin independent, it does not contribute to $\delta$ which only depends on EC and VC. As the $e-h$ separation increases, the $\mathrm{VC}$ decreases while the $\mathrm{EC}$ increases as a consequence of a larger exciton size that is implied by the increase of the overlap between excitons. Therefore, for moderate densities there is, for a critical value $d_{\mathrm{cr}} \simeq 0.12 a \simeq$ $10 \AA$, a change of sign in the measurable quantity $\delta$. Within our numerical precision, this critical separation turns out to be independent on $P$. For $d<d_{\mathrm{cr}} \mathrm{VC}$ dominates on EC giving $\delta>0$ while for $d>d_{\mathrm{cr}} \mathrm{EC}$ dominates and $\delta<0$. It must be stressed that the value of $d_{\mathrm{cr}}$ is quite low and the change of sign of the splitting effect could be observable, for example, applying moderately high external electric fields.

Figure 2 shows the phase diagram of the sign of $\delta$ in terms of the physical magnitudes of interest in the problem: $n a^{2}$ and $d / a$. For high $d$ or $n$ the kinetic energy dominates the $e-h$ attraction and the $e$ - $h$ pairs are not bound having a positive chemical potential.

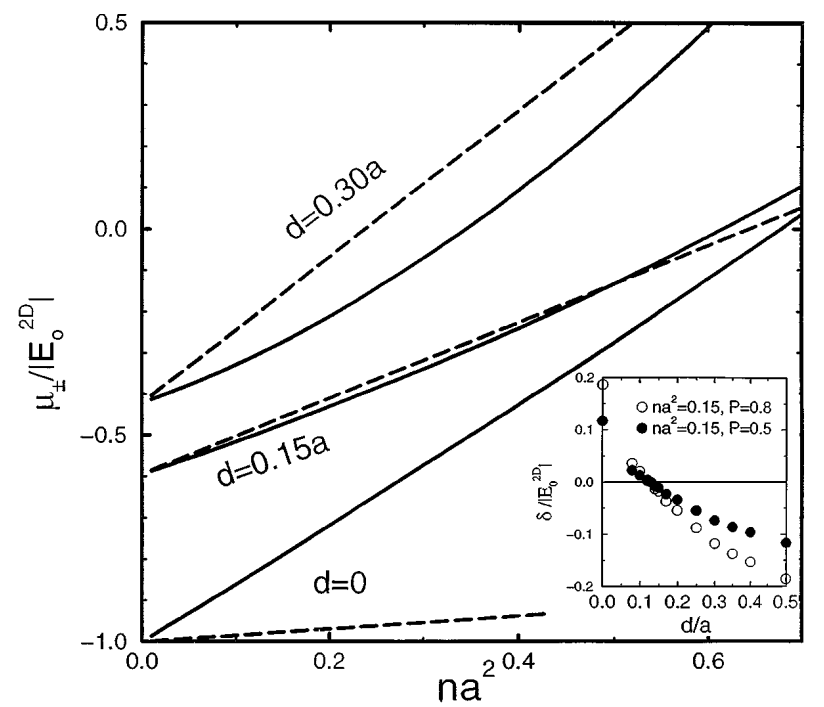

FIG. 1. Majority $\mu_{+}$(continuous lines) and minority $\mu_{-}$ (dashed lines) chemical potentials as a function of the density $n$ for $d=0.0,0.15$, and 0.30 . The inset shows the splitting $\delta=\mu_{+}-\mu_{-}$as a function of the $e-h$ separation $d$ for $n a^{2}=0.15$ and $P=0.5$ and 0.8 . 


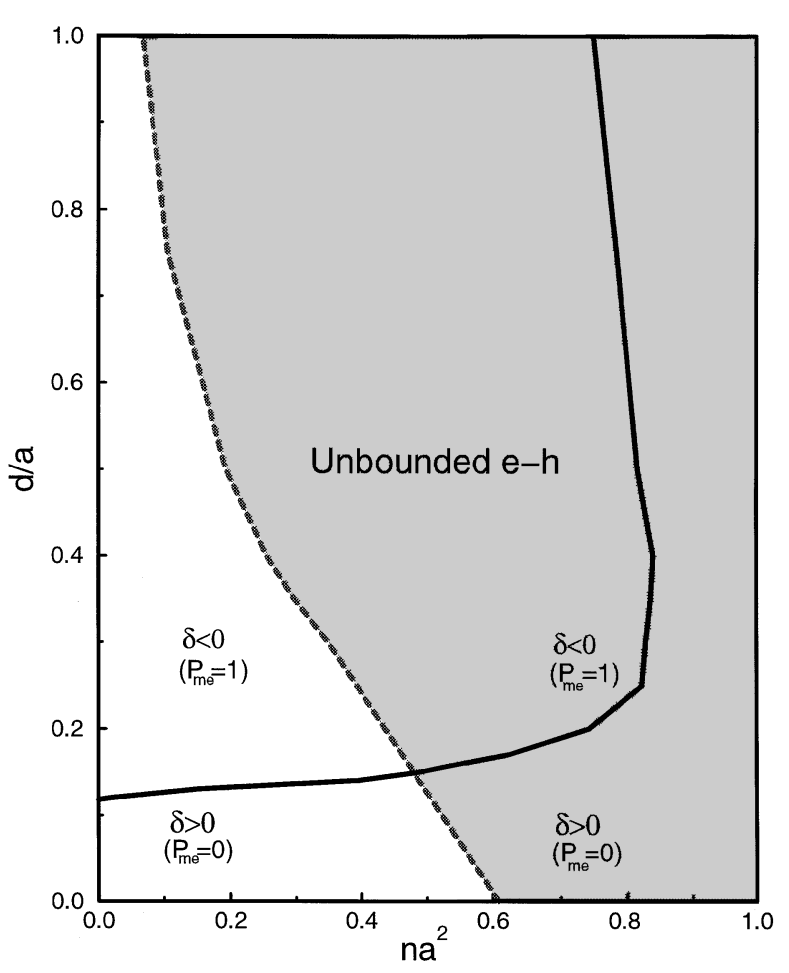

FIG. 2. Phase diagram for both the sign of $\delta=\mu_{+}-\mu_{-}$ and the polarization $P_{m e}$ of the condensate (see text). Shaded region corresponds to a condensate of unbounded $e-h$ pairs.

Therefore, experimental determination of the splitting must be performed in the more accessible region of low $d$ and $n$ where excitons are well defined. The important point is that the diagram also gives information about the polarization of the condensate. This is easily understood by realizing that the derivative of the total energy per pair $E$, in the thermodynamic limit, is

$$
\left(\frac{\partial E}{\partial P}\right)_{N} \equiv E\left(N_{+}+1, N_{-} 1\right)-E\left(N_{+}, N_{-}\right)=\delta .
$$

Since for fixed $d$ and $n, \delta$ does not change sign when $P$ varies, the derivative (7) is a monotonous function of $P$. The region of the diagram with positive splitting corresponds to an increase of the total energy $E$ with $P$, i.e., the system has minimum energy when it is unpolarized, $P_{m e}=0$. However, in the region with $\delta<0$, the derivative (7) is also negative indicating that the system should have minimum energy when completely polarized, $P_{\mathrm{me}}=1$. For $d<d_{\mathrm{cr}} \mathrm{VC}$ always dominates and the unpolarized state $\delta>0$ has the minimum energy. For increasing separation $d$, the excitons are less strongly bound and EC dominates allowing the appearance of a polarized phase with $\delta<0$. It is difficult to predict whether, for adequate values of $d$ and $n$, the spins of the condensate would spontaneously polarize or not because this process should occur in the presence of spin relaxation mechanisms that we have not considered, as mentioned above. Therefore, the best way of experimentally analyzing the phase diagram is to measure the sign of $\delta$ instead of looking for magnetic properties. The sign of $\delta$ would remain as given by Fig. 2 even if other physical contributions were to be included in our analysis. Available experiments $[12,13]$ are for $d=0$ and, in the whole range of experimentally accessible densities, always $\delta$ results to be positive as it happens in our phase diagram.

In summary, we have studied a condensate of $2 \mathrm{D}$ excitons having different spin orientations. We have shown that the spin degree of freedom plays a very important and nontrivial role in exciton condensation. We have analyzed the case of resonant excitation finding that the ground state energy depends strongly on spin polarization. When electron and hole components are separated from each other, the relative importance of exchange and vertex correction interactions can be altered. This causes a change of sign in $\delta$. This splitting between the chemical potentials of different exciton components can be measured by time-resolved spectroscopy $[12,13]$. The sign of $\delta$ is related to the minimum energy configuration of the condensate, i.e., spin polarized or spin unpolarized. All these features are shown and discussed in a phase diagram in $n$ and $d$.

We thank L. Viña and L. Brey for useful discussions. This work was supported in part by CICYT of Spain under Contract No. MAT 94-0982-C02-01 and by the Comunidad Autonoma de Madrid under Contract No. AE00330/95.

[1] The third component of the angular momentum $(M)$ is usually denoted in the field as the spin.

[2] A. J. Leggett, Rev. Mod. Phys. 47, 331 (1975).

[3] L. V. Keldysh and Yu. V. Kopaev, Fiz. Tverd. Tela 6, 2791 (1964) [Sov. Phys. Solid State 6, 2219 (1965)].

[4] D. Jerome et al., Phys. Rev. 158, 462 (1967); B. I. Halperin and T.M. Rice, Solid State Physics (Academic, New York, 1968), Vol. 21, p. 115; R. Zimmermann, Phys. Status Solidi B 76, 191 (1976).

[5] C. Comte and P. Nozières, J. Phys. (Paris) 43, 1069 (1982); P. Nozières and C. Comte, ibid. 43, 1083 (1982).

[6] H. Haug et al., Prog. Quantum Electron. 9, 3 (1984).

[7] C. Comte and G. Mahler, Phys. Rev. B 34, 7164 (1986).

[8] X. Zhu et al., Phys. Rev. Lett. 74, 1633 (1995).

[9] X. Naveh et al., Phys. Rev. Lett. 77, 900 (1996); T. Portengen et al., Phys. Rev. Lett. 76, 3384 (1996); H. Chu and Y. C. Chang, Phys. Rev. B 54, 5020 (1996).

[10] G. Mahan, Many Particle Physics (Plenum, New York, 1982).

[11] Along this paper we take $N_{+} \geq N_{-}$.

[12] T. C. Damen et al., Phys. Rev. Lett. 67, 3432 (1991).

[13] L. Viña et al., Phys. Rev. B 54, R8317 (1996).

[14] J. Shah, Hot Carriers in Semiconductor Nanostructures (Academic, San Diego, 1992).

[15] J. Fernández-Rossier and C. Tejedor (unpublished).

[16] M.Z. Maialle et al., Phys. Rev. B 47, 15776 (1993).

[17] J. Fernández-Rossier et al., Phys. Rev. B 54, 11582 (1996). 